УДК 519.21

А. А. Дороговцев (Ин-т математикн НАН Украяны, Киев)

\title{
МЕРЫ ПОСЕПЕНИЯ И ЭРГОДИЧЕСКАЯ ТЕОРЕМА \\ ДЛЯ ПОСЛЕДОВАТЕЛЬНОСТИ ИТЕРАЦИЙ СО СЛУЧАЙНЫМИ ВОЗМУЩЕНИЯМИ
}

By using local visitation measures, we describe the limit behavior of a sequence of iterations with random unequally distributed perturbations. As a corollary, we obtain a version of the local ergodic theorem.

За допомогою локальних мір перебування описано граничну поведінку послідовності ітерацін з внпадковими не однахово розподіленимн збуреннямн. Як наслідок отримано варіант локальної ергодичної теореми.

1. Меры посешения. В данной работе исследуется поведение рекуррентных последовательностей вида

$$
x_{n+1}=\varphi\left(x_{n}\right)+\xi_{n}, \quad n \geq 0, \quad x_{0} \in \mathbb{R}^{d} .
$$

Здесь $\varphi: \mathbb{R}^{d} \rightarrow \mathbb{R}^{d}$ - непрерывная функция, $\left\{\xi_{n} ; n \geq 0\right\}$ - последовательность независимых случайных векторов в $\mathbb{R}^{d}$, заданных на вероятностном пространстве $(\Omega, \mathcal{F}, P)$. Для последовательности (1) множество частичных пределов и частота посещения их окрестностей могут быть описаны в терминах мер посещения, введенных в [1]. Поэтому этот пункт содержит определения и факты из [1], необходимые в дальнейшем.

Пусть $\left\{u_{n} ; n \geq 0\right\}-$ детерминированная последовательность элементов из $\mathbb{R}^{d}$.

Определение 1. Считающей мерой последовательности $\left\{u_{n} ; n \geq 0\right\}$ для $n \geq 0$ называется льера

$$
v_{n}=\sum_{k=0}^{n} \delta_{u_{k}}
$$

zде $\delta_{u}$ - мера единичной льассы, сосредоточенная в точке $и$.

Определение 2. Последовательность конечных мер $\left\{\mu_{n} ; n \geq 0\right\}$ на $\sigma$ алгебре борелевских льножеств пространства $\mathbb{R}^{d}$ называется возрастающей, если

1) для произвольного борелевского $A \subset \mathbb{R}^{d}$ последовательность $\left\{\mu_{n}(A)\right.$; $n \geq 0\}$ не убывает,

2) $\lim _{n \rightarrow \infty} \mu_{n}\left(\mathbb{R}^{d}\right)=+\infty$.

Определение 3. Точка $u \in \mathbb{R}^{d}$ называется точкой роста возрастающей последовательности мер $\left\{\mu_{n} ; n \geq 0\right\}$, если $\forall \varepsilon>0$ :

$$
\lim _{n \rightarrow \infty} \mu_{n}(B(u, \varepsilon))=+\infty \text {. }
$$

Здесь $B(u, \varepsilon)$-открытый шар радиуса $\varepsilon$ с иентром в $и$.

Множество всех точек роста возрастающей последовательности мер $\left\{\mu_{n}\right.$; $n \geq 0\}$ обозначим $\mathfrak{W}_{\vec{\mu}}$. Это замкнутое множество. Если $\left\{v_{n} ; n \geq 0\right\}-$ пос- 
ледовательность считающих мер последовательности $\left\{u_{n} ; n \geq 0\right\}$, то $\mathfrak{W}_{\bar{v}}$ множество частичных пределов $\left\{u_{n} ; n \geq 0\right\}$.

Определение 4. Две последовательности возрастающих мер $\left\{\mu_{n} ; n \geq 0\right\}$ $u\left\{\lambda_{n} ; n \geq 0\right\}$ эквивалентны, если

1) $\mathfrak{M}_{\bar{\mu}}=\mathfrak{M}_{\bar{\lambda}}=\mathfrak{M} \neq \emptyset$,

2) для любых ограниченных открытьх множестө $U_{1}{ }_{1}$ и $U_{2}$ таких, что замыкание $\bar{U}_{1} \subset U_{2}, U_{1} \cap \mathfrak{M} \neq \emptyset$,

$$
\varliminf_{n \rightarrow \infty} \frac{\mu_{n}\left(U_{2}\right)}{\lambda_{n}\left(U_{1}\right)} \geq 1, \quad \varliminf_{n \rightarrow \infty} \frac{\lambda_{n}\left(U_{2}\right)}{\mu_{n}\left(U_{1}\right)} \geq 1 .
$$

Определение 5. Последовательность лер, эквивалентная последовательности считающих мер последовательности $\left\{u_{n} ; n \geq 0\right\}$, называетсл последовательностью мер посещения $\left\{u_{n} ; n \geq 0\right\}$.

В [1] доказаны следуюиие утверждения.

Теорема 1. Если возрастающая последовательность мер $\left\{\mu_{n} ; n \geq 0\right\}$ эквивалентна последовательности $\left\{c_{n} \lambda ; n \geq 0\right\}$, где $\left\{c_{n} ; n \geq 0\right\}$ - числовал последовательность, а $\lambda$-веролтностнал мера на $\mathbb{R}^{d}$, то меры $\left\{\mu_{n} / c_{n}\right.$; $n \geq 0\}$ сходятсл слабо $\kappa \lambda$.

Теорема 2. Пусть $\left\{\eta_{n} ; n \geq 0\right\}$-ограниченная последовательность независильх случайных векторов в $\mathbb{R}^{d}$. Тогда существует нножество $\Omega_{0} \subset \Omega$ такое, ито $P\left(\Omega_{0}\right)=1$ идля любого $\omega \in \Omega_{0}$ последоеательность

$$
\left\{\sum_{i=0}^{n} P \eta_{i}^{(-1)} ; n \geq 0\right\}
$$

является последовательностью мер посещения для $\left\{\eta_{n}(\omega) ; n \geq 0\right\}$.

Теорема 2 может быть интерпретнрована следующим образом. С вероятность 1 последовательность $\left\{\eta_{n} ; n \geq 0\right\}$ имеет постоянное множество частичных пределов и частота посещения окрестностей частичных пределов описывается мерами (2). В некоторых случаях утверждения теорем 1 и 2 можно комбинироватъ, что приводит $\mathrm{k}$ эргодической теореме для исходной случайной последовательности (не обязательно состоящей из независимых случайных величнн [2]). Цель данной статьи - получить аналог теоремы 2 для последовательости (1). Для этого нам понадобится дополнительное определение.

Определение 4 очевидным образом распространяется на случай $\sigma$-конечных мер $\left\{\mu_{n} ; n \geq 1\right\}$ и $\left\{\lambda_{n} ; n \geq 1\right\}$, сужения которых на каждый шар конечны.

Определение 5. Возрастающал последовательность мер (возможно б-конечных) эквивалентна последовательности считающих мер последовательности $\left\{u_{n} ; n \geq 1\right\} \subset \mathbb{R}^{d}$ называетсл последовательностью локальных мер посещения последовательности $\left\{u_{n} ; n \geq 1\right\}$.

2. Меры посещения для последовательности итерацй. Предположим, что функщия $\varphi$ из (1) такова, что

$$
\exists \alpha \in(0 ; 1): \forall x, y \in \mathbb{R}^{d}:\|\varphi(x)-\varphi(y)\| \leq \alpha\|x-y\| .
$$

Пусть случайные векторы $\left\{\xi_{n} ; n \geq 0\right\}$ имекот распределення абсолютно непрерывные относитељно меры Лебега с плотностями $\left\{p_{n} ; n \geq 1\right\}$. 
Теорема 3. Пусть существует борелевская функция $p: \mathbb{R}^{d} \rightarrow(0 ;+\infty)$, для которой

1) существует сходящаяся $\kappa+\infty$ последовательность $\left\{a_{n} ; n \geq 1\right\}$ такая, что $\forall n \geq 1$ :

$$
\begin{aligned}
& 0<\alpha_{n}=\inf \left\{\frac{p_{n}(x)}{p(x)}:\|x\| \leq a_{n}\right\} \leq \\
& \leq \beta_{n}=\sup \left\{\frac{p_{n}(x)}{p(x)}:\|x\| \leq a_{n}\right\}<+\infty ;
\end{aligned}
$$

2) $\alpha_{n} \rightarrow 0, n \rightarrow \infty, \alpha_{n}-\beta_{n}, n \rightarrow \infty$;

3) $\forall m \geq 1, r=0,1, \ldots, m-1$ :

$$
\sum_{k=1}^{\infty} \alpha_{k m+r}=+\infty
$$

4) $\lim _{\|x\| \rightarrow+\infty} \varphi(x)=u_{0}$.

Тогда существует льножество $\Omega_{0}$ полной вероятности такое, что для любого $\omega \in \Omega_{0}$ последовательность $\left\{x_{n}(\omega) ; n \geq 0\right\}$ имеет в качестве последовательности локальных мер посещения последовательность $\left\{\gamma_{n} \sigma ; n \geq\right.$ $\geq 0\}$, гәе

$$
\gamma_{n}=\sum_{j=1}^{n} \alpha_{j}, \quad n \geq 1, \quad d \sigma=p\left(x-u_{0}\right) d x .
$$

Доказательство. Для каждого $m \geq 1$ определим последовательность $\left\{x_{n}^{m} ; n \geq m\right\}$ следующим образом:

$$
x_{n}^{m}=\xi_{n}+\varphi\left(\xi_{n-1}+\varphi\left(\xi_{n-2}+\ldots+\varphi\left(\xi_{n-m+1}+\varphi(0)\right)\right) \ldots\right) .
$$

Поскольку функция $\varphi$ удовлетворяет условию Липшица с $\alpha<1$ и ограничена, то

$$
\lim _{m \rightarrow \infty} \varlimsup_{n \rightarrow \infty}\left\|x_{n}-x_{n}^{m}\right\|=0(\bmod P) .
$$

Построим вначале последовательность локальных мер посещения для последовательности $\left\{x_{n}^{m} ; n \geq m\right\}$ при фиксированном $m$. Рассмотрим для $r=0$, $1, \ldots, m-1$ последовательность $\left\{x_{j m+r}^{m} ; j \geq 1\right\}$. Согласно (3) это последовательность незавнсимых случайых элементов в $\mathbb{R}^{d}$. Следовательно, аналогично теореме $2,\left\{x_{j m+r}^{m} ; j \geq 1\right\}$ на множестве полнон вероятности имеет своен последовательностью мер посещений последовательность сумм распределенин вида

$$
\sum_{j=1}^{n} P\left(x_{j m+r}^{m}\right)^{(-1)}, \quad n \geq 1
$$

Для того чтобы исследовать асимттотическое поведение последовательности мер (4), рассмотрим отдельное слагаемое $P\left(x_{n}^{m}\right)^{(-1)}$ при $n \rightarrow \infty$. Согласно определению 5 достаточно исследовать поведение $P\left\{x_{n}^{m} \in \Delta\right\}$ для отхрытых 
ограниченных $\Delta$. Обозначнм через $v_{n}^{m}$ распределение $x_{n}^{m}-\xi_{n}$. Тогда для достаточно больших номеров $n$

$$
\begin{gathered}
P\left\{x_{n}^{m} \in \Delta\right\}=\iint_{\mathbb{R}^{d}} \int_{\Delta} p_{n}(y-x) d y v_{n}^{m}(d x) \geq \\
\geq \alpha_{n} \iint_{\mathbb{R}^{d}} p_{\Delta}(y-x) d y v_{n}^{m}(d x), \\
P\left\{x_{n}^{m} \in \Delta\right\} \leq \beta_{n} \int_{\mathbb{R}^{d}} \int_{\Delta} p_{n}(y-x) d y v_{n}^{m}(d x) .
\end{gathered}
$$

Поскольку согласно условию $\lim _{n \rightarrow \infty} \beta_{n}=0$, то

$$
P-\lim _{n \rightarrow \infty}\left\|\xi_{n}\right\|=+\infty \text {. }
$$

Поэтому при $m \geq 1$

$$
P-\lim _{n \rightarrow \infty} \varphi\left(x_{n}^{m}\right)=P-\lim _{n \rightarrow \infty} \varphi\left(\xi_{n-1}^{m}+\varphi\left(x_{n-1}^{m-1}\right)\right)=u_{0} .
$$

Вследствие локальной интегрируемости функции $p$ и ограниченности $\Delta$ фунхщия

$$
\mathbb{R}^{d} \ni x \mapsto \int_{\Delta} p(y-x) d y
$$

является непрерывнон. Поэтому

$$
P\left\{x_{n}^{m} \in \Delta\right\}-\alpha_{n} \int_{\Delta} p\left(y-u_{0}\right) d y, \quad n \rightarrow \infty .
$$

Согласно лемме Штольца

$$
\sum_{j=1}^{n} P\left(x_{j m+r}^{m}\right)^{(-1)}(\Delta)-\sum_{j=1}^{n} \alpha_{j m+r} \int_{\Delta} p\left(y-u_{0}\right) d y, \quad n \rightarrow \infty .
$$

Поэтому аналогично [1] для последовательности $\left\{x_{j m+r}^{m} ; j \geq 1\right\}$ последовательность мер

$$
\left\{\sum_{j=1}^{n} a_{j m+r} \sigma ; n \geq 1\right\}
$$

является последовательностью локальных мер посещения на множестве полной вероятности $\Omega_{r}$. Пусть $\omega \in \bigcap_{r=0}^{m-1} \Omega_{r}, U_{1}$ и $U_{2}$ - непустые открытые ограниченные множества такие, что замыкание $\bar{U}_{1}$ лежит в $U_{2}$. Тогда

$\forall \varepsilon>0 \quad \exists n_{0}: \forall n \geq n_{0}:$

$$
\begin{gathered}
\sum_{k=1}^{n} \chi_{U_{2}}\left(x_{k}^{m}(\omega)\right)=\sum_{r=0}^{m-1} \sum_{k=r(\bmod m), k \leq n} \chi_{U_{2}}\left(x_{k}^{m}(\omega)\right) \geq \\
\geq(1-\varepsilon) \sum_{r=0}^{m-1} \sum_{k \text { m } r(\bmod m), k \leq n} \alpha_{k} \sigma\left(U_{1}\right)=(1-\varepsilon) \gamma_{n} \sigma\left(U_{1}\right), \\
\sum_{k=1}^{n} \chi_{U_{1}}\left(x_{k}^{m}(\omega)\right) \leq(1+\varepsilon) \gamma_{n} \sigma\left(U_{2}\right) .
\end{gathered}
$$


Следовательно, на множестве полной вероятности последовательность мер $\left\{\gamma_{n} \sigma ; n \geq 1\right\}$ является последовательностью локальных мер посещения для $\left\{x_{n}^{m} ; n \geq 1\right\}$. Для завершения доказательства теоремы осталось воспользоватъся тем, что

$$
\lim _{m \rightarrow \infty} \varlimsup_{n \rightarrow \infty}\left\|x_{n}-x_{n}^{m}\right\|=0(\bmod P)
$$

аналогично тому, как это делалось в [1]. Теорема доказана.

Следствие (локальная эргодическая теорема). При выполнении условий теоремы 3 для любой непрерывной функиии $f: \mathbb{R}^{d} \rightarrow \mathbb{R}$ с компактным носителехь справедливо равенство

$$
\lim _{n \rightarrow \infty} \frac{1}{\gamma_{n}} \sum_{k=1}^{n} f\left(x_{k}\right)=\int_{\mathbb{R}^{d}} f(y) \sigma(d y) \quad(\bmod P) .
$$

Замечание. Условие существования предела $\lim _{|x| \rightarrow+\infty}=u_{0}$ можно заменить совместным условием на $\varphi$ и последовательность $\left\{\xi_{n} ; n \geq 1\right\}$, гарантируюшим слабую сходимость мер $\left\{v_{n}^{m} ; n \geq 1\right\}$ к пределу, не зависящему от $m$ и имеющему компактный носитель. Кроме того, при $p \equiv 1$ надобность в условия 4 естественно отпадает и его можно заменить требованием ограниченности функцин $\varphi$.

Пример. Пусть $\left\{\eta_{n} ; n \geq 1\right\}$ - последовательность независимых одинаково распределенных гауссовских случайных величин со средним 0 и дисперсией 1 . Рассмотрим последовательность итеращий

$$
x_{n+1}=\frac{1}{2} \sin x_{n}+n \eta_{n}, \quad n \geq 1, \quad x_{1}=0 .
$$

Для случаћ̆ных величин $\xi_{n}=n \eta_{n}, n \geq 1$, выполнены условия теоремы с функцией $p \equiv 1, a_{n}=n^{1 / 4}, \alpha_{n}=\frac{1}{\sqrt{2 \pi n}} \exp \left(-\frac{1}{2 \sqrt{n}}\right), n \geq 1$. Следователњно, последовательность итераций $\left\{x_{n} ; n \geq 1\right\}$ на множестве полной вероятности имеет в качестве локальных мер посещения последовательность $\frac{\ln n}{\sqrt{2 \pi}} \lambda, n \geq 2$, где $\lambda$ - мера Лебега. В частности, для произвольной финитной непрерывной функции $f$ с вероятностью 1 справедливо равенство

$$
\lim _{n \rightarrow \infty} \frac{\sqrt{2 \pi}}{\ln n} f\left(x_{k}\right)=\int_{\mathbb{R}} f(x) d x .
$$

1. Дороговцев А. А. Некоторые характеристики последовательностей итеращий со случайными возмущениями // Укр. мат. журн. - 1996. - 48, № 8. - C. 1047 - 1063.

2. Dorogovtsev A. A., Denisievskii N. A. Path-wise behavior of stationary sequences // Theor. Stochast. Processes. - 1996. - 2 (18), N N 3-4. - P. 17-26.

Получено 22.05.97 